\title{
Dominant Epistasis Between Two Quantitative Trait Loci Governing Sporulation Efficiency in Yeast Saccharomyces cerevisiae
}

\author{
Juraj Bergman ${ }^{1}$, Petar T. Mitrikeski ${ }^{1,2}$ and Krunoslav Brčić-Kostić1* \\ ${ }^{1}$ Laboratory for Evolutionary Genetics, Division of Molecular Biology, Ruđer Bošković Institute, \\ Bijenička cesta 54, HR-10000 Zagreb, Croatia \\ ${ }^{2}$ Institute for Research and Development of Sustainable Ecosystems, Faculty of Veterinary Medicine, \\ Heinzelova 55, HR-10000 Zagreb, Croatia
}

Received: November 18, 2014

Accepted: July 3, 2015

\begin{abstract}
Summary
Sporulation efficiency in the yeast Saccharomyces cerevisiae is a well-established model for studying quantitative traits. A variety of genes and nucleotides causing different sporulation efficiencies in laboratory, as well as in wild strains, has already been extensively characterised (mainly by reciprocal hemizygosity analysis and nucleotide exchange methods). We applied a different strategy in order to analyze the variation in sporulation efficiency of laboratory yeast strains. Coupling classical quantitative genetic analysis with simulations of phenotypic distributions (a method we call phenotype modelling) enabled us to obtain a detailed picture of the quantitative trait loci (QTLs) relationships underlying the phenotypic variation of this trait. Using this approach, we were able to uncover a dominant epistatic inheritance of loci governing the phenotype. Moreover, a molecular analysis of known causative quantitative trait genes and nucleotides allowed for the detection of novel alleles, potentially responsible for the observed phenotypic variation. Based on the molecular data, we hypothesise that the observed dominant epistatic relationship could be caused by the interaction of multiple quantitative trait nucleotides distributed across a 60-kb QTL region located on chromosome XIV and the RME1 locus on chromosome VII. Furthermore, we propose a model of molecular pathways which possibly underlie the phenotypic variation of this trait.
\end{abstract}

Key words: budding yeast, sporulation inheritance, two-locus epistasis, QTL analysis, phenotype simulation

\section{Introduction}

Phenotypic variation of complex, quantitative traits is an important subject of research in modern biology and one particular model for investigating such traits is sporulation efficiency in the yeast Sacharomyces cerevisiae. Yeast cells undergo sporulation when environmental con- ditions are not suitable for mitotic growth due to nitrogen starvation and/or a change in cellular respiration, caused by both an absence of a fermentable carbon source and the presence of a nonfermentable carbon source, such as acetate (1). This two-staged process, consisting of meiosis and spore morphogenesis, results in four spores encapsu-

\footnotetext{
*Corresponding author: Phone: +385 (0)1 457 1285; E-mail: brcic@irb.hr Contributions: This study was suggested by KBK, and further developed by KBK and PTM. Experimental design is by PTM and KBK. All the experiments were executed by JB. The computational analysis was suggested and designed by JB, as well as the molecular model. The manuscript was conceived and written by JB, PTM and KBK
} 
lated in an ascus, which allows the yeast cells to withstand a variety of environmental stresses (2). The proportion of cells capable of completing the process of sporulation, in a given time period, varies among different wild yeast strains (3-5) as well as among laboratory strains $(6,7)$. The quantitative features of sporulation efficiency have been reported extensively and it is generally acknowledged that this trait serves as an efficient model for quantitative genetics study (3-7). Most studies of sporulation efficiency are based on quantitative trait loci (QTLs) mapping and detection of differences at the gene and nucleotide levels between high- and low-sporulating strains (5-7). The genotype-phenotype correlation is usually tested via functional analysis generally comprising of reciprocal hemizygosity analysis (RHA), allelic exchange methods and site-directed mutagenesis (5-7). Using these approaches, Deutschbauer et al. (6) managed to pinpoint three nucleotide variants (associated with genes RME1, MKT1 and TAO3) which account for $>90 \%$ of the difference in sporulation between the low-sporulating strain S288c and high-sporulating strain SK1 of S. cerevisiae. In another study (7), by analysing the same two strains, additional nucleotide variants associated with sporulation efficiency were identified. Yet another source of variation in sporulation efficiency comes from the study of natural yeast populations (5). In total, allelic variants of nine genes (RME1, TAO3, MKT1, RAS2, PMS1, SWS2, FKH2, IME1 and RSF1) and their impact on the trait have been described in these studies. Therefore, the abundance of identified variants provides an excellent framework for correlating sporulation efficiencies of different yeast strains with specific gene and nucleotide compositions of the QTLs detected by these studies.

Since the number and compositions of QTLs underlying sporulation efficiency have already been subject to comprehensive analysis (5-7), the focus of this study is to investigate sporulation efficiency from a fresh perspective, detecting and defining complex interrelations between the detected QTLs. Thus, by combining computational and molecular approaches with a classical quantitative genetic approach ('six generation' crossing strategy) we provide novel insights into the interactions between the QTLs underlying this trait.

\section{Materials and Methods}

\section{Yeast strains}

The first set of parental strains of Saccharomyces cerevisiae used in this study were SK1 and S288c strains. The SK1 parental strain was generated by mating the isogenic haploid SK1 strains (ho::LYS2, lys2, ura3, leu2::hisG, trp1: :his $G$ and his3::hisG), while the S288c parental strain was generated by mating two isogenic BY4743 strains: segregant 2a (MATa, his3- $\Delta$, leu2 $\Delta 0$, met15 $\Delta 0$ and ura3 $\Delta 0)$ and segregant 2c (MAT $\alpha$, his3- $\Delta$, leu $2 \Delta 0$, lys $2 \Delta 0$ and ura3 $\Delta 0)$. The second set of parental strains was the isogenic W303 constructed by mating two haploid W303 BMA 64 strains (ura3-1, trp1 , leu2-3,112, his3-11 and ade2-1) and the isogenic AA1973 constructed by mating strains 181026 (MATd, leu2, ura3, trp1, cyh2 and arg4-RV) and 181028 (MATa, leu2, ura3, trp1, cyh2 and arg4-Bg). The $\mathrm{F}_{2}$ genera- tions were constructed by randomly coupling spores isolated from the $\mathrm{F}_{1}$ strains, while the $\mathrm{Bc}_{1.1}$ and $\mathrm{Bc}_{1.2}$ backcross generations were constructed by randomly coupling the $\mathrm{F}_{1}$ spores with the $\mathrm{P}_{1}$ and $\mathrm{P}_{2}$ spores.

\section{Media}

Yeast cells were grown on yeast extract-peptone-glucose (YPG) plates and liquid YPG medium, both of which had the same composition (in $\mathrm{g} / \mathrm{L}$ ): yeast extract 10 , bacto peptone 20 and glucose 20 (the YPG plates additionally contained $20 \mathrm{~g} / \mathrm{L}$ of agar). Yeast extract and peptone were purchased from Difco Laboratories Inc., Detroit, MI, USA, glucose from Kemika d.d., Zagreb, Croatia, and agar from Sigma-Aldrich, St. Louis, MO, USA. Sporulation was induced on plates containing potassium acetate $(5 \mathrm{~g} / \mathrm{L}$; Sigma-Aldrich), a solution of supplementary amino acids (Sigma-Aldrich) and nucleotides $(8.3 \mathrm{~mL} / \mathrm{L}$; Sigma-Aldrich) (8) and agar (20 g/L). A volume of $5 \mathrm{~mL}$ of the sporulation medium was used per Petri dish in order to ensure a thin and homogenous layer of the medium allowing for easier cell microscopy and manipulation.

\section{Construction of the six basic generations}

The foundation of this study was a construction of six basic generations, most often applied when studying plant quantitative traits $(9,10)$. These six generations are derived from two inbred parental lines $\left(\mathrm{P}_{1}\right.$ and $\mathrm{P}_{2}$ generations), which significantly differ in their phenotypic value, and then mated to produce four additional strains: the $\mathrm{F}_{1}\left(\mathrm{P}_{1} \times \mathrm{P}_{2}\right)$ and $\mathrm{F}_{2}\left(\mathrm{~F}_{1} \times \mathrm{F}_{1}\right)$ generations, as well as two backcross generations: $\mathrm{Bc}_{1.1}\left(\mathrm{P}_{1} \times \mathrm{F}_{1}\right)$ and $\mathrm{Bc}_{1.2}\left(\mathrm{P}_{2} \times \mathrm{F}_{1}\right)$. Similar methods (sometimes referred to as Birmingham style quantitative genetics), although with different goals, have already been applied to different fungal species (11-13).

The six basic generations (strains) were derived from two isogenic yeast strains $\left(\mathrm{P}_{1}\right.$ and $\left.\mathrm{P}_{2}\right)$ which differed significantly in their phenotypic value. For the construction of the $\mathrm{F}_{2}$ and the backcross $\mathrm{Bc}_{1.1}$ and $\mathrm{Bc}_{1.2}$ generations, it was first necessary to sporulate the $F_{1}$ strains. After being incubated for $24 \mathrm{~h}$ on sporulation medium, the $\mathrm{F}_{1}$ cells were transferred into $100-\mu \mathrm{L}$ digestion solution (containing $96 \mu \mathrm{L}$ of sterile $\mathrm{H}_{2} \mathrm{O}, 1.5 \mu \mathrm{L}$ of Zymolyase ${ }^{\circledR} 20 \mathrm{~T}(10 \mathrm{mg} /$ $\mathrm{mL}$; Seikagaku Corporation, Tokyo, Japan) and $2.5 \mu \mathrm{L}$ of $0.5 \mathrm{M}$ dichlorodiphenyltrichloroethane (DDT; Sigma-Aldrich). The suspension was then incubated for $30 \mathrm{~min}$ at $37{ }^{\circ} \mathrm{C}$. After incubation, the suspension was transferred onto a YPG plate and the haploid spores were then isolated from the asci using a micromanipulator. The $\mathrm{F}_{2}$ strains were constructed by randomly intercrossing $\mathrm{F}_{1}$ spores, while the $\mathrm{Bc}_{1.1}$ and $\mathrm{Bc}_{1.2}$ generations were constructed by randomly crossing $\mathrm{F}_{1}$ spores with $\mathrm{P}_{1}$ and $\mathrm{P}_{2}$ spores.

\section{Determination of sporulation efficiency}

The mean value and variance of sporulation efficiency for each of the six generations were determined under constant experimental conditions. Sporulation efficiency of one colony was used as a basic unit for calculating the mean value and variance of sporulation efficiency for a particular generation. In order to determine the sporulation efficiency of one colony, the colony was first trans- 
ferred from the YPG plate to $10 \mathrm{~mL}$ of liquid YPG medium. The cells were incubated for $24 \mathrm{~h}$ at $29^{\circ} \mathrm{C}$. After $24 \mathrm{~h}$, $5 \mathrm{~mL}$ of the cell suspension were centrifuged at $1968 \times g$ for $5 \mathrm{~min}$ at $4{ }^{\circ} \mathrm{C}$. The supernatant was discarded and the cell pellet was resuspended in $10 \mathrm{~mL}$ of sterile $\mathrm{H}_{2} \mathrm{O}$. The centrifugation and resuspension steps were repeated to ensure that the medium was thoroughly removed. Next, 10$-\mu \mathrm{L}$ droplets of the cell suspension were inoculated onto sporulation plates and left to sporulate for $24 \mathrm{~h}$ at $30^{\circ} \mathrm{C}$. The sporulation efficiency of the colony was determined by using a microscope to count approx. 300-350 cells and record the number of asci among the counted cells. The result was expressed as a percentage of sporulated cells. At least 50 colonies of SK1/S288c crosses and 100 colonies of W303/AA1973 crosses were analysed per generation to provide an accurate estimate of the mean phenotype and variance.

\section{Quantitative genetic analysis of the six basic generations}

The broad $\left(H^{2}\right)$ and narrow $\left(h^{2}\right)$ sense heritabilities were calculated using standard formulae (9). The expected mean phenotypic values, under the assumption of the three-parameter $(\mathrm{m}=$ midparent value, $[\mathrm{a}]=$ additive genetic effect and $[\mathrm{d}]=$ dominance genetic effect) additive-dominance (AD) model of inheritance, were calculated in order to test the adequacy of the model using the A, B and C scaling tests (9). Furthermore, to test various types of epistasis, the AD model was expanded to include additional digenic interaction parameters ([aa]=additive $\times$ additive effect, $[\mathrm{ad}]=$ additive $\times$ dominance effect and $[\mathrm{dd}]=$ dominancexdominance effect) (9). The significance of each parameter was tested using a $t$-test and when a parameter proved to be insignificant, the remaining parameters were reestimated by the weighted least squares procedure (9).

\section{Computational analysis of the six basic generations}

The main goal of the phenotype modelling method developed for this study was to generate theoretical phenotypic distributions which correspond to specific inheritance models and are comparable to the observed experimental distributions. The six generation experimental data supplied information about inter- and intra-QTL interactions providing the basic assumptions needed to generate these theoretical distributions. The theoretical phenotypic distributions were simulated using $\mathrm{R}$ software (14) which requires the estimation of three parameters: theoretical phenotypic ratios, mean values and variances of phenotypic classes present in a particular theoretical distribution.

For the construction of the theoretical $\mathrm{F}_{2}$ and $\mathrm{Bc}_{1.2}$ generations several assumptions were made. Firstly, it was assumed that only dominant alleles were responsible for higher sporulation efficiencies and that these alleles were present exclusively in the high-sporulating parental strain $\left(\mathrm{P}_{1}\right)$, while the recessive, low-sporulating versions of these alleles were present in the low-sporulating parental strain $\left(\mathrm{P}_{2}\right)$. Secondly, it was assumed that the variance caused by experimental conditions had an equal effect on all phenotypic values. In other words, complete dominance and equal environmental variance were supposed. Two types of theoretical distributions (AD and dominant epistatic (DE) distributions) were constructed.
Taking into account the previous assumptions, an AD distribution comprised a certain number of phenotypic classes (depending on the number of independently segregating loci) whose mean phenotypic values are equidistantly distributed across the phenotypic value range. The extreme values of this range were equated with the experimental mean phenotypic values of the parental generations $\left(\bar{P}_{1}\right.$ and $\left.\bar{P}_{2}\right)$. Also, for the equidistant property to apply, locus contributions to the overall mean phenotypic value of a particular generation were assumed to be equal. Therefore, the mean phenotypic values of the AD phenotypic classes $\left(\bar{P}_{\mathrm{N}, \mathrm{r}}\right)$ present in a particular theoretical $\mathrm{AD}-\mathrm{F}_{2}$ generation were determined by the formula:

$$
\bar{P}_{\mathrm{N}, \mathrm{r}}=\bar{P}_{2}+\frac{\left(\bar{P}_{1}-\bar{P}_{2}\right) \cdot(N-r)}{N}
$$

where $N$ is the number of independently segregating loci, $r$ is an integer which depends on $N$ (ranging from 0 to $N$ ) and determines the number of phenotypic classes present in a particular $\mathrm{AD}-\mathrm{F}_{2}$ generation. The ratio of phenotypic classes can be calculated using binomial expansion (15). The variances of the extreme phenotypic classes $\left(\bar{P}_{1}\right.$ and $\bar{P}_{2}$ phenotypes) present in a theoretical generation were equated with the corresponding experimental variances, while the variances of the intermediary phenotypic classes were equated with the environmental variance $\mathrm{V}_{\mathrm{E}}$ (calculated as the mean of the phenotypic variances of $\mathrm{P}_{1}, \mathrm{P}_{2}$ and $\mathrm{F}_{1}$ experimental generations; 9 ). These variances allowed for the generation of Gaussian distributions (using the 'rnorm' function in R) around the mean phenotypic values of different phenotypic classes present in a particular theoretical generation, and when the corresponding frequencies were applied to these distributions, together they constituted a phenotypic distribution of the corresponding theoretical generation.

When constructing the distributions of the theoretical DE generations it was assumed that, since epistasis is present, at least two loci contributed to the phenotypic variation with only one locus being epistatic and the rest of the loci being hypostatic. The effect of the epistatic locus was such that when at least one dominant allele was present at that locus, the phenotypic effects of all other loci were suppressed. In other words, when the epistatic locus was homozygous or heterozygous for the dominant allele, only the dominant $\mathrm{P}_{1}$ phenotype was expressed. The extreme phenotypic values in the DE generations were equated with the corresponding mean experimental values $\left(\bar{P}_{1}\right.$ and $\left.\bar{P}_{2}\right)$, while the mean phenotypic value of the cumulative intermediary DE phenotype was equated with the mean experimental intermediary phenotype $\left(\bar{P}_{\text {int }}\right)$, calculated as the mean of the phenotypic values of the experimental $\mathrm{F}_{2}$ colonies which did not fall in the range of the observed $\mathrm{P}_{1}$ or $\mathrm{P}_{2}$ phenotype. Again, the theoretical $\mathrm{F}_{2}$ frequencies of the phenotypic classes present in the DE generations were calculated using adjusted formulae based on binomial expansion (15).

The phenotypic variances of each of the classes present in a particular theoretical generation were determined as with the AD generations, and the construction of theoretical distributions was also conducted in the previously described manner. The theoretical backcross generations were constructed in a similar way, with the phenotypic frequencies adjusted to match the specific phenotypic ratios of these generations. 
Different theoretical phenotypic ratios corresponded to different trait inheritance models, making possible the testing of multiple inheritance scenarios. These scenarios were tested by comparing phenotypically heterogeneous generations $\left(\mathrm{F}_{2}\right.$ and $\left.\mathrm{Bc}_{1.2}\right)$ with the corresponding theoretical distributions. The $t$-test was used to compare the overall mean phenotypic values, the $\chi^{2}$ test to compare phenotypic ratios and the Kolmogorov-Smirnov test to test the equality of the distributions.

\section{DNA sequencing of sporulation loci}

Genes that were previously reported to differ at a nucleotide level between high- and low-sporulating strains (5-7) were PCR-amplified from the parental W303 and AA1973 strains and sequenced. The sequencing was done at Macrogen Europe (Amsterdam, The Netherlands) using PCR sequencing primers (Table 1) designed by the software Web Primer (16). The sequences were assembled with the DNA Baser Sequence Assembler software (17) and the analysis of the assembled sequences was conducted using a custom Python script (18).

\section{Results}

\section{Quantitative genetic analysis}

The applicability of the six generation approach was originally tested for SK1/S288c crosses (Fig. S1 and Table
S1; available at www.ftb.com.hr). The outcome of this analysis was an AD inheritance model of sporulation efficiency (Table S2; available at www.ftb.com.hr), which is in accordance with previously reported results (6). Therefore, applying the six generation experimental design, we proceeded to analyse the W303/AA1973 crosses (the $P_{1}$, $\mathrm{P}_{2}, \mathrm{~F}_{1}, \mathrm{~F}_{2} \mathrm{Bc}_{1.1}$ and $\mathrm{Bc}_{1.2}$ generations discussed in further text refer to these crosses). Phenotypic distributions of the six W303/AA1973 basic generations are shown in Fig. 1. By careful analysis of these distributions, three distinct phenotypic classes can be distinguished: the high-sporulating W303, the low-sporulating AA1973 and an intermediary phenotypic class centred at around $83 \%$ sporulation efficiency (present in the $\mathrm{F}_{2}$ and $\mathrm{Bc}_{1,2}$ generations). Sporulation efficiencies of at least one hundred colonies per generation were determined, from which the mean values and variances of sporulation efficiencies were calculated accordingly (Table 2 ). $H^{2}$ was calculated to be approx. 0.99 , while $h^{2}$ was approx. 0 .

The initial model used to test trait inheritance was a basic AD model which assumes that the phenotypic value of the quantitative trait is the exclusive result of additive and dominance effects within the loci, as well as of additive effects between the loci affecting the trait. The results of the A, B and C scaling tests (see Materials and Methods) are presented in Table 3 . The inadequacy of the AD inheritance model demanded an expansion of the three-parameter model to a six-parameter model of inheritance

Table 1. PCR primers used in this study

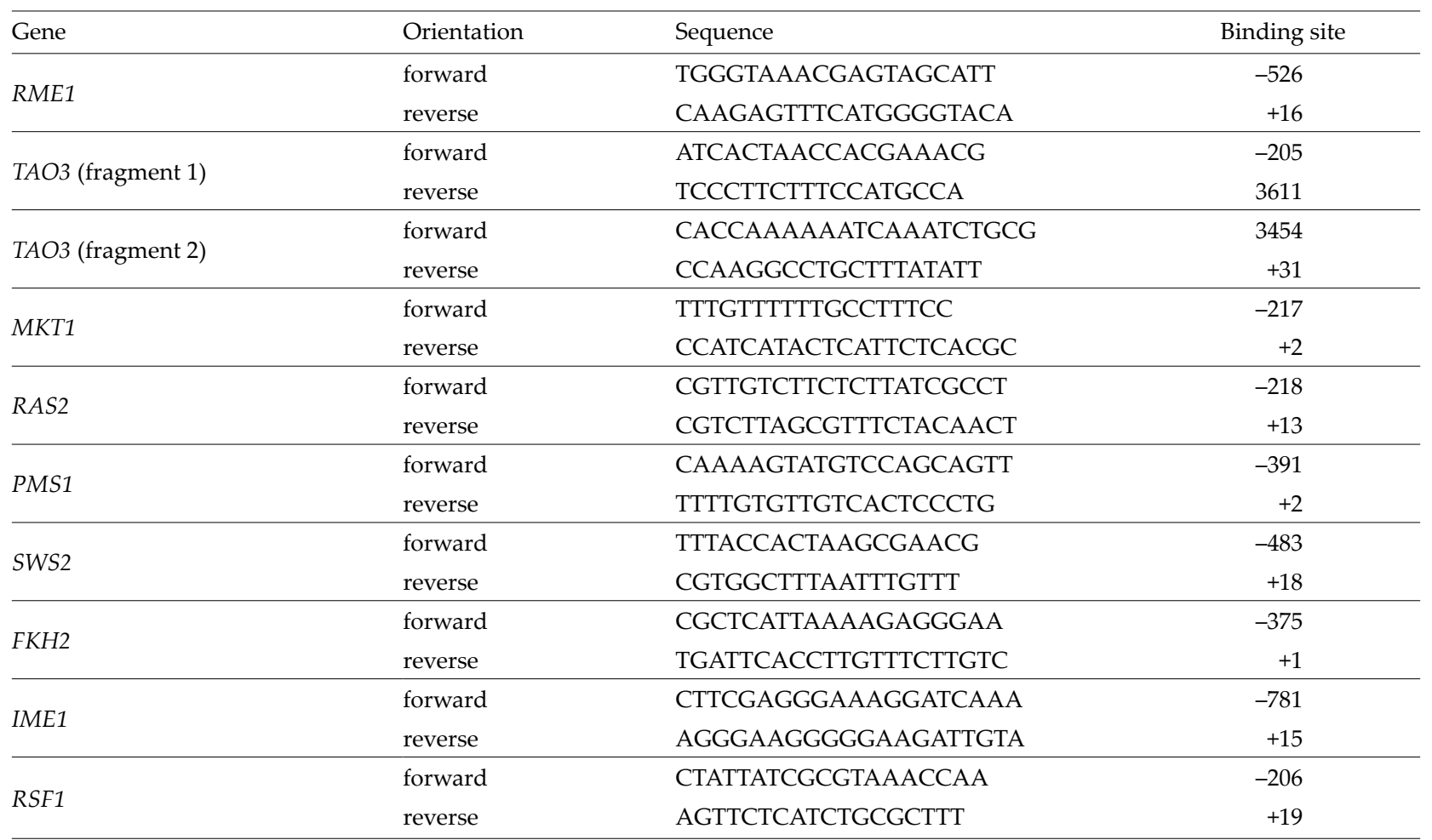

$-=$ denotes the downstream position of the primer binding the site relative to the start codon

$+=$ denotes the upstream position of the primer binding the site relative to the stop codon

Note: Due to its length, the TAO3 gene was sequenced in two overlapping fragments; therefore, the reverse primer of fragment 1 and the forward primer of fragment 2 do not contain prefixes because they are located within the gene itself (the binding sites of these primers denote the positions within the gene relative to the start of the coding region) 

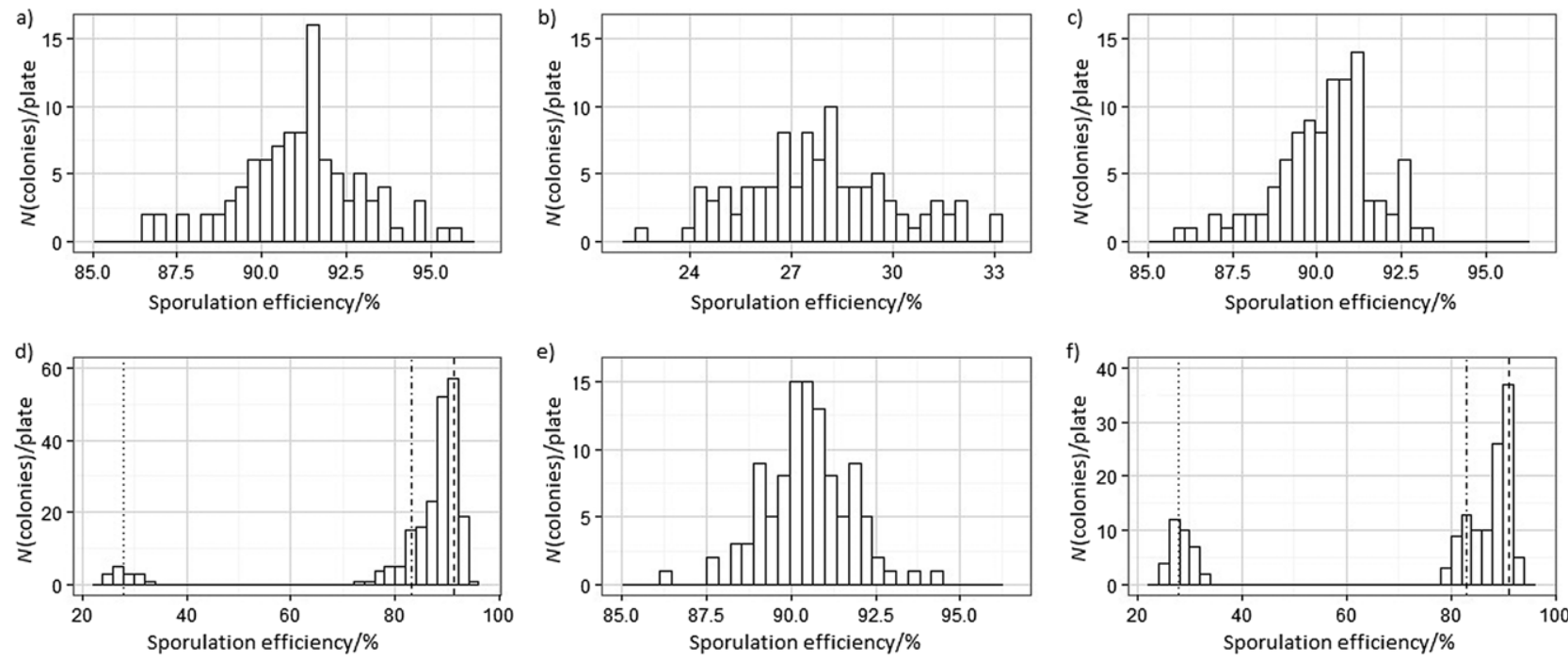

Fig. 1. Phenotypic distributions of the six basic W303/AA1973 generations: a-c) phenotypic distributions of the non-segregating $P_{1}$ (W303), $\mathrm{P}_{2}$ (AA1973) and $\mathrm{F}_{1}$ generations, respectively; $\mathrm{d}-\mathrm{f}$ ) distributions of the segregating $\mathrm{F}_{2}, \mathrm{Bc}_{1.1}$ and $\mathrm{Bc}_{1.2}$ generations, respectively. The phenotypic ranges of the $\mathrm{F}_{2}$ and $\mathrm{Bc}_{1.2}$ generations are much wider than those of the non-segregating and $\mathrm{Bc}_{1.1}$ generations, indicating a heterogeneous composition in phenotypes present in these generations. The dotted, dash-dotted and dashed vertical lines in d) and f) represent the mean phenotypic values of the $P_{1}$, intermediary and $P_{2}$ phenotypes present in the $F_{2}$ and $\mathrm{Bc}_{1.2}$ generations, respectively

Table 2. The mean values and variances of sporulation efficiencies of the six basic W303/AA1973 generations

\begin{tabular}{lcccr}
\hline Generation & Cross & $N$ & $\begin{array}{c}\text { Mean } \\
\text { value } \%\end{array}$ & Variance \\
\hline $\mathrm{P}_{1}$ (W303) & - & 100 & 91.12 & 3.33 \\
$\mathrm{P}_{2}$ (AA1973) & - & 100 & 27.96 & 5.40 \\
$\mathrm{~F}_{1}$ & $\mathrm{P}_{1} \times \mathrm{P}_{2}$ & 100 & 90.23 & 2.03 \\
$\mathrm{~F}_{2}$ & $\mathrm{~F}_{1} \times \mathrm{F}_{1}$ & 214 & 83.92 & 250.67 \\
$\mathrm{Bc}_{1.1}$ & $\mathrm{P}_{1} \times \mathrm{F}_{1}$ & 106 & 90.48 & 1.59 \\
$\mathrm{Bc}_{1.2}$ & $\mathrm{P}_{2} \times \mathrm{F}_{1}$ & 148 & 73.71 & 647.64 \\
\hline
\end{tabular}

$N=$ number of observations

Table 3. Adequacy of the additive dominance (AD) model of inheritance tested by A, B and C scaling tests (9)

\begin{tabular}{ccccr}
\hline Test & Result & $\begin{array}{c}\text { Degree of } \\
\text { freedom }\end{array}$ & t-test & p-value \\
\hline A & -0.43 & 303 & -1.276 & 0.203 \\
B & 29.23 & 345 & 6.972 & $<0.001$ \\
C & 36.14 & 510 & 8.311 & $<0.001$ \\
\hline
\end{tabular}

$\mathrm{p}>0.05$ indicates that the difference is not statistically significant, i.e. the correctness of the model

which included the effects of various types of epistasis. Only two additional digenic interaction parameters, i.e. [ad] and [dd] proved to have a significant effect on the overall mean phenotypic values of the six generations, which resulted in a five-parameter model of inheritance (Table 4). Moreover, this model of inheritance with the additional [ad] and [dd] parameters provided the best fit to the experimental mean phenotypic values. These re-
Table 4. Five-parameter inheritance model (9) of the W303/ AA1973 crosses

\begin{tabular}{crcrc}
\hline Parameter & $\begin{array}{c}\text { Estimated } \\
\text { value } \%\end{array}$ & $\begin{array}{c}\text { Degree of } \\
\text { freedom }\end{array}$ & t-test & p-value \\
\hline $\mathrm{m}$ & $60.05 \pm 0.6$ & 663 & 95.318 & $<0.001$ \\
[a] & $31.3 \pm 0.6$ & 198 & 50.468 & $<0.001$ \\
[d] & $61.6 \pm 13$ & 762 & 4.790 & $<0.001$ \\
[ad] & $-33.5 \pm 13$ & 450 & -2.610 & $<0.001$ \\
[dd] & $-31.4 \pm 13$ & 762 & -2.449 & 0.015 \\
\hline
\end{tabular}

*Values are expressed as mean \pm standard deviation $p>0.05$ indicates that the parameter value is not significantly different from zero

$\mathrm{m}=$ midparent value, $[\mathrm{a}]=$ additive genetic effect, $[\mathrm{d}]=$ dominance genetic effect, [ad]=additive $\times$ dominance effect,

[dd] $=$ dominance $\times$ dominance effect

sults are in accordance with the observed narrow-sense heritability and indicate a possible DE relationship between QTLs affecting the trait.

\section{Phenotype modelling}

The small number of phenotypic classes present in the experimental distributions (Fig. 1) indicated that a small number of loci most likely underlie the experimental W303/AA1973 phenotypic distributions. Therefore, the phenotype modelling methodology was utilised to predict the exact number of QTLs affecting the trait and the types of interactions between them. This was achieved by comparing the experimental phenotypic distributions of the six basic generations with theoretical simulations of phenotypic distributions which included genetic effects such as additivity, dominance and epistasis. Two inheritance models were considered: the basic AD and the DE models. 
In order to test the $\mathrm{AD}$ model of inheritance, four $\mathrm{AD}$ $-\mathrm{F}_{2}$ theoretical distributions (each assuming the independent segregation of a different number of loci, ranging from one to four) were constructed and compared with the experimental $\mathrm{F}_{2}$ generation (Fig. 2). Again, the results of the statistical tests showed that the AD model is inadequate when explaining the experimental phenotypic distributions of the six generations (Table 5).

Having again excluded the AD model, we proceeded with the testing of DE models. Two types of dominant epistasis tested were the duplicate dominant epistasis and the single dominant epistasis. Duplicate epistasis was excluded as a plausible explanation of inheritance (Fig. S2; available at www.ftb.com.hr), while the single dominant epistasis model provided the best fit to experimental data, as described further in the text. We constructed two theoretical DE- $\mathrm{F}_{2}$ distributions and each was compared with the experimental $\mathrm{F}_{2}$ distribution (Figs. 3a and $b$ ); the first DE- $\mathrm{F}_{2}$ distribution (DE1) assumed the independent segregation of two loci while the second distribution (DE2) assumed the segregation of three loci. The DE2 model failed all statistical tests, while the $t$-test and the $\chi^{2}$ test for the DE1 model showed that there was no significant difference between the experimental and theoretical $\mathrm{F}_{2}$ distributions (Table 5). Although the p-value of the Kolmogorov-Smirnov D-statistic test was the highest compared to the rest of the distributions, the difference between the experimental $\mathrm{F}_{2}$ distribution and the DE1 theoretical distribution was still statistically significant. Nevertheless, the two-locus DE1 model provided the best fit of all the tested theoretical generations. Besides the $\mathrm{F}_{2}$ distribution, the phenotypic distribution of the $\mathrm{Bc}_{1.2}$ generation was the only other distribution to exhibit significant phenotypic variance. Therefore, in order to further investigate the adequacy of the DE models of inheritance, theoretical DE$\mathrm{Bc}_{1.2}$ generations were constructed and compared with the experimental $\mathrm{Bc}_{1.2}$ generation (Figs. $3 \mathrm{c}$ and d). Again, sta-
Table 5. Comparison of theoretical and experimental phenotypic distributions of W303/AA1974 crosses

\begin{tabular}{ccccc}
\hline $\begin{array}{c}\text { Inheritance } \\
\text { model }\end{array}$ & Generation & $\begin{array}{c}\text { p-value } \\
(t \text {-test })\end{array}$ & $\begin{array}{c}\text { p-value } \\
\left(\chi^{2} \text { test }\right)\end{array}$ & $\begin{array}{c}\text { p-value } \\
\text { (KS-test })\end{array}$ \\
\hline AD1 & $\mathrm{F}_{2}$ & $<0.001$ & - & $<0.001$ \\
$\mathrm{AD} 2$ & $\mathrm{~F}_{2}$ & $<0.001$ & $<0.001$ & $<0.001$ \\
$\mathrm{AD} 3$ & $\mathrm{~F}_{2}$ & $<0.001$ & $<0.001$ & $<0.001$ \\
$\mathrm{AD} 4$ & $\mathrm{~F}_{2}$ & $<0.001$ & - & $<0.001$ \\
$\mathrm{DE} 1$ & $\mathrm{~F}_{2}$ & 0.255 & 0.361 & 0.001 \\
$\mathrm{DE} 2$ & $\mathrm{~F}_{2}$ & $<0.001$ & $<0.001$ & $<0.001$ \\
$\mathrm{DE} 1$ & $\mathrm{Bc}_{1.2}$ & 0.937 & 0.806 & 0.004 \\
$\mathrm{DE} 2$ & $\mathrm{Bc}_{1.2}$ & $<0.001$ & $<0.001$ & $<0.001$ \\
\hline
\end{tabular}

$\mathrm{p}>0.05$ indicates that the difference is not statistically significant, i.e. the correctness of the model

$\mathrm{KS}=$ Kolmogorov-Smirnov test

Note: In the case of AD1, there are no intermediary phenotypes present, so the frequencies of the intermediary phenotypes (present in the experimental distribution) cannot be compared. The same applies to the AD4, in which there are no AA1973 phenotypes present

tistical tests were applied and the two-locus DE1 model proved once more as the most plausible solution for the inheritance of sporulation efficiency (Table 5). These results are in accordance with the five-parameter model of inheritance (Table 4) and the theoretical DE phenotypic ratios of the $F_{2}$ and $B_{1.2}$ generations (12:3:1 and 2:1:1, respectively).

\section{Molecular analysis of sporulation loci}

The goal of the molecular analysis was to find two candidate QTLs (predicted by phenotype modelling) which could possibly be responsible for the DE phenotypic rela-
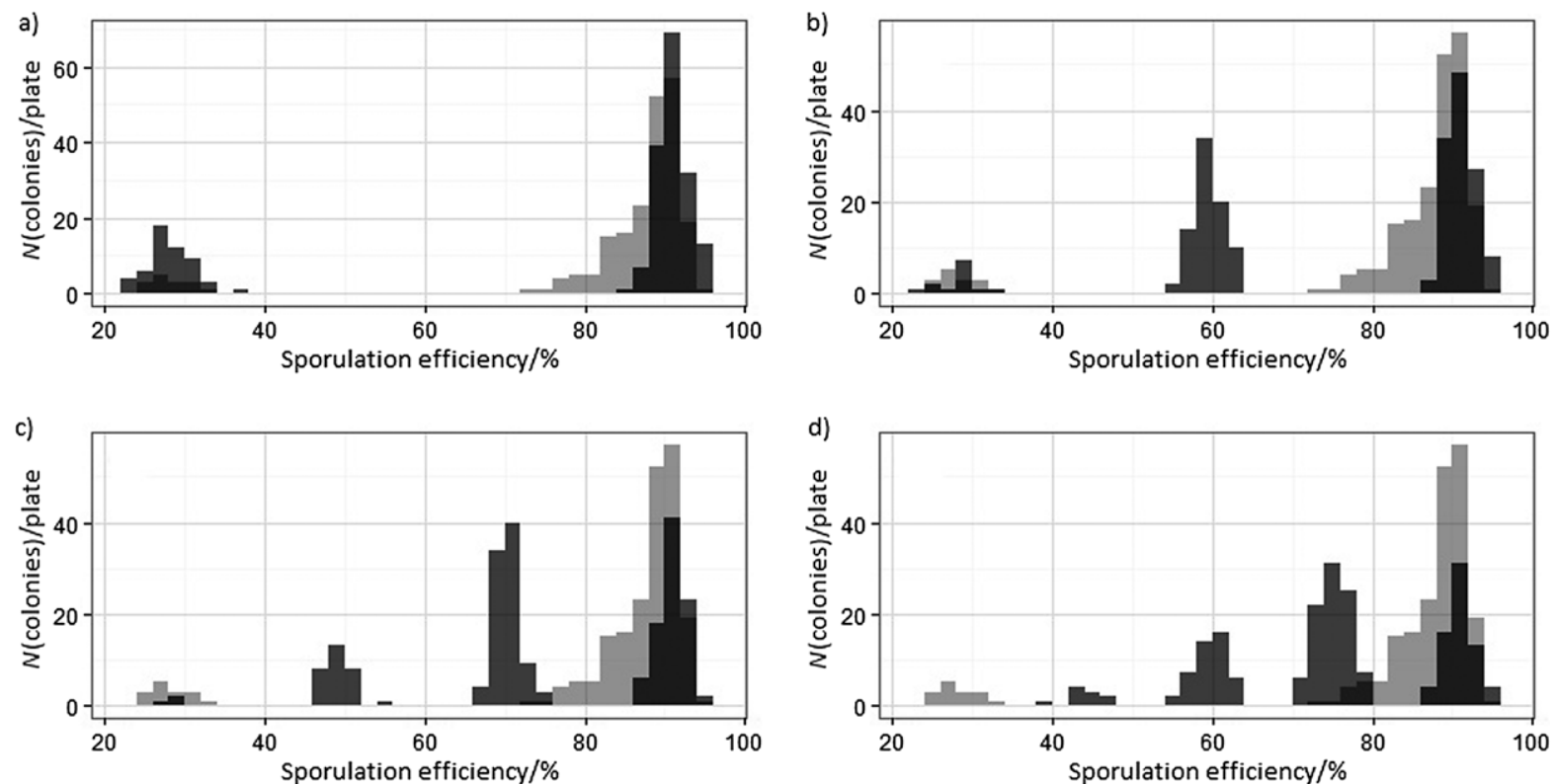

Fig. 2. Comparison of the experimental $F_{2}$ distribution with the theoretical AD- $F_{2}$ distributions in W303/AA1973 crosses: a-d) the overlapping of the experimental $\mathrm{F}_{2}$ distribution with the one-, two-, three- and four-locus AD- $\mathrm{F}_{2}$ theoretical distributions, respectively. The light grey areas represent the experimental $F_{2}$ distribution, the dark grey areas represent the theoretical AD- $F_{2}$ distributions and the black areas represent the overlapping of these distributions 

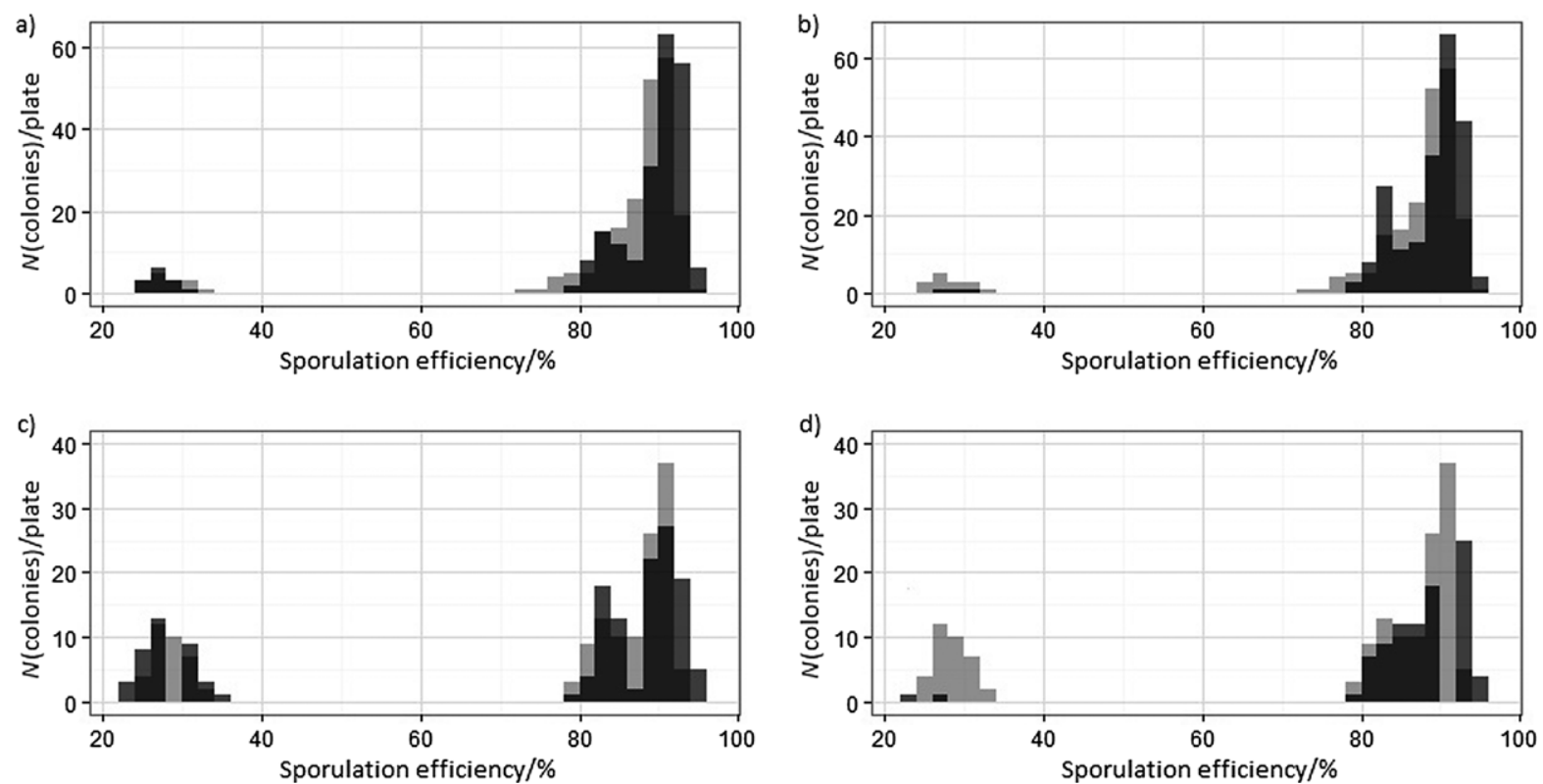

Fig. 3. Comparison of experimental and theoretical dominant epistatic (DE) phenotypic distributions in W303/AA1973 crosses: a) and $\mathrm{b}$ ) the overlapping of the experimental $\mathrm{F}_{2}$ distribution with the two- and three-locus DE- $\mathrm{F}_{2}$ theoretical distributions, respectively; c) and d) the overlapping of the experimental $\mathrm{Bc}_{1.2}$ distribution with the two- and three-locus DE-Bc $\mathrm{C}_{1.2}$ theoretical distributions, respectively. The light grey areas represent the experimental phenotypic distributions, the dark grey areas represent the theoretical DE distributions and the black areas represent the overlapping of these distributions

tionship observed experimentally. Therefore, the parental W303/AA1973 strains were sequenced for the nine genes that had previously been reported to have an impact on sporulation efficiency (RME1, TAO3, MKT1, RAS2, PMS1, SWS2, FKH2, IME1 and RSF1; 5-7). Out of the nine analysed loci, four were excluded as candidate genes in the DE model of inheritance. The IME1 gene on chromosome $X$ and the RAS2 gene on chromosome XIV did not differ in the nucleotide sequence between the parental strains, while the RSF1 gene on chromosome XIII differed in one silent polymorphism. Also, the TAO3 gene on chromosome IX of both parental strains contained insertions which resulted in a truncated Tao3 protein (the W303 gene had insertion 6865_6866insT and the AA1973 gene had insertion 5254_5255ins A). Because of this fact, it is unlikely that TAO3 plays a role in determining the difference in sporulation efficiency of the W303 and AA1973 strains. Consequently, we turned our attention to the five remaining loci that contained coding mutations and were distributed across chromosomes XIV and VII. A comprehensive list of these mutations, which are assumed to have an effect on sporulation efficiency, is presented in Table 6. Taking these results into account, the detected two-locus DE relationship most likely reflects the interaction between the 60-kb QTL region on chromosome XIV and the RME1 gene on chromosome VII.

\section{Discussion}

Quantitative genetic analysis supplemented by molecular and computational methods

This investigation aimed to provide insights into the genetic interactions and functions of previously detected loci shown to govern sporulation efficiency in yeast (5-7).
Table 6. Polymorphisms assumed to affect sporulation efficiency in the W303 and AA1973 strains

\begin{tabular}{lcccc}
\hline Locus & Chromosome & Position & $\begin{array}{c}\text { W303 } \\
\text { nucleotide }\end{array}$ & $\begin{array}{c}\text { AA1973 } \\
\text { nucleotide }\end{array}$ \\
\hline RME1 & VII & -308 & deletion & A \\
& & $570 \_571^{*}$ & insertion of T & - \\
MKT1 & XIV & 89 & $\mathrm{G}(\mathrm{G})$ & $\mathrm{A}(\mathrm{D})$ \\
PMS1 & XIV & $1515^{*}$ & $\mathrm{~T}(\mathrm{D})$ & $\mathrm{A}(\mathrm{E})$ \\
SWS2 & XIV & $-91 \_-90$ & - & insertion of A \\
FKH2 & XIV & $121^{*}$ & $\mathrm{C}(\mathrm{L})$ & T (F) \\
\hline
\end{tabular}

*a newly detected polymorphism

Note: The letters in parentheses indicate the amino acid encoded by the particular nucleotide change

Extensive genetic mapping of these QTLs provided by these studies (5-7) enabled us to focus on developing a model of inheritance for this trait. Therefore, the goal of this study was to explore yeast sporulation efficiency by combining classical quantitative genetic analysis with computational and molecular approaches.

The six generation experimental design provided an excellent starting point as demonstrated by the results of the SK1/S288c crosses (Fig. S1 and Tables S1 and S2; available at www.ftb.com.hr). Also, the utilization of this experimental design for sporulation efficiency analysis is further justified when it is taken into consideration that each observation is actually a mean value of sporulation efficiency of a particular colony, providing statistically more powerful results (when compared to most plant 
quantitative trait analyses). The proposed $\mathrm{AD}$ inheritance model corroborates the previously detected additive effects between loci responsible for the difference in phenotypic value between the SK1/S288c strains (6). Since these strains were already molecularly characterised in relation to sporulation efficiency $(6,7)$, the main focus of this study were the six generation crosses of the W303 and AA1973 strains which provided a fresh template for the analysis of sporulation efficiency. The fact that the difference in mean sporulation efficiency between these parental strains was large $(>60 \%)$ and that the environmental variance of the trait was low $(<6)$ indicated that an allelic diversity among QTLs was responsible for the difference in sporulation efficiency. Furthermore, the sporulation efficiencies of the $\mathrm{P}_{1}, \mathrm{~F}_{1}$ and $\mathrm{Bc}_{1.1}$ strains had almost identical mean phenotypic values and variances, indicating that the loci that contribute to higher sporulation efficiency are dominant and present within the W303 genome, while their low sporulation counterparts are all present within the AA1973 genome. The value of $H^{2}$ indicated a low impact of the experimental conditions on the total phenotypic variation, i.e. the genotypic composition of the trait almost entirely accounted for the observed phenotypic variation, while $h^{2}$ showed that the additive effects between loci affecting sporulation efficiency were nonexistent. Moreover, a computational method of phenotype modelling which was developed for this study, in combination with multiple statistical tests, provided a powerful tool in determining the number and the genetic relationships of QTLs affecting sporulation efficiency. It is important to note that this method was successfully applied because, unlike the majority of quantitative traits, a small number of large-effect QTLs governs sporulation efficiency $(6,7)$, which in turn results in distinct phenotypic classes that can be used as starting points for modelling phenotypic distributions. Also, the molecular analysis of candidate QTLs helped to pinpoint the molecular architecture of these loci. Such a combined approach (comprising quantitative genetic, computational and molecular analysis) enabled a thorough understanding of the inheritance of sporulation efficiency. The initial model used to test the inheritance was a basic AD model which was expanded to include epistatic effects. In order to test inheritance models, scaling tests were applied (9) and theoretical phenotypic distributions were constructed and compared with experimental data. The main reason for excluding duplicate epistasis (Fig. S2; available at www. $\mathrm{ftb} . c 0 \mathrm{~m} . \mathrm{hr}$ ) as a plausible inheritance model was the presence of an intermediary phenotype centred at around 83 $\%$ sporulation efficiency, whose phenotypic range partially overlapped that of the $P_{1}$ strain (Figs. 1d and f). Taking into account this information, single dominant epistasis, as opposed to duplicate dominant epistasis, became the most plausible explanation of trait inheritance.

\section{QTLs affecting sporulation efficiency}

Molecular analysis of previously reported loci that affect sporulation efficiency (5-7) provided the evidence that a DE relationship between the 60-kb QTL region on chromosome XIV and the RME1 gene on chromosome VII could possibly explain the experimental phenotypic distributions of the six generations of the W303/AA1973 cross.
The upstream region of the repressor of meiosis, the RME1 gene, on chromosome VII of the high-sporulating W303 strain contained the deletion -308delA, while the low-sporulating AA1973 strain did not contain this mutation. This deletion is assumed to be correlated with low sporulation efficiency (6). However, the RME1 coding sequence of the W303 strain contained an insertion (570_571insT) which results in the truncation of the Rme1 zinc finger binding domains (19) most likely rendering the Rme1 protein non-functional. Chromosome XIV has a 60-kb QTL (7), which contains five of the nine analysed genes (MKT1, RAS2, PMS1, SWS2 and FKH2). All of these genes (except RAS2) showed nucleotide variation between the two parental strains. The MKT1 gene differed between the parental strains in one silent and two coding mutations (G30D and R453K). The same two coding mutations had previously been detected as variations in the MKT1 gene between the high-sporulating SK1 strain and the low-sporulating S288c strain. The G30D mutation was confirmed to be a quantitative trait nucleotide (6). The PMS1 gene differed between the W303 and AA1973 strains in one silent and one coding mutation (D505E). This coding change of aspartic acid into glutamic acid should not have a significant effect on the function of the Pms1 protein because of the similar properties of these two amino acids. The SWS2 gene differed between parental strains in one silent and one coding mutation (L41F). Also, an insertion of an adenine in the upstream region of the AA1973 SWS2 gene (-91_-90insA) corresponds to the same insertion in the low-sporulating S288c strain (7). FKH2 gene of the AA1973 strain has a deletion in the coding sequence (118delC) truncating the AA1973 Fkh2 protein. The described allelic specificity of the W303 and AA1973 strains, comprising of novel as well as previously detected polymorphisms (Table 6), may be the molecular cause of the observed DE QTL relationship.

Based on our results, we propose that the high sporulation QTL on chromosome XIV of the W303 strain act like an epistatic locus because of the abundance of genes which promote high sporulation. Even when this QTL is heterozygous at the W303 allele and the RME1 locus is homozygous at the AA1973 allele, the sporulation efficiency will remain high because of the strong sporulationpromoting effects of the W303 alleles on chromosome $X I V$. The observed 12:3:1 phenotypic ratio of the $F_{2}$ generation supports this hypothesis. The truncated AA1973 Fkh2 protein, as well as the low-sporulating versions of the MKT1 and SWS2 AA1973 genes on chromosome XIV may be the main cause of the low sporulation efficiency of this strain. The W303 Rme1 protein has mutated in such a way that most of its zinc finger-binding domains (19) are truncated so it is most likely unable to bind the promoter region of the initiator of meiosis, the IME1 gene, while the non-truncated AA1973 Rme1 protein probably still retains a certain level of repressor function. Therefore, in this scenario, the W303 RME1 allele promotes high sporulation efficiency, while the AA1973 counterpart favours low sporulation efficiency. Furthermore, the fact that the RME1 locus of the low-sporulating AA1973 strain, analysed in this study, did not contain the -308ins A mutation, partially responsible for the low sporulation phenotype of the S288c strain (6), can explain the higher sporulation of the AA1973 strain (approx. $28 \%$ ) when compared with 
the observed S288c sporulation efficiency (approx. $9 \%$ ). The sporulation efficiencies of the high-sporulating strains (W303 and SK1) are basically identical (approx. 91 $\%)$, showing that different combinations of nucleotides can yield very similar phenotypes.

The efficiency of the W303 QTL on chromosome XIV to initiate and successfully execute the sporulation process could be such that a heterozygous version of this QTL is sufficient for yeast cells to achieve the high sporulation phenotype. In other words, the W303 QTL of chromosome XIV achieves complete dominance over the AA1973 QTL of chromosome XIV and also has an epistatic effect over the RME1 gene on chromosome VII (masking the phenotypic effect of this locus), which results in the observed DE relationship. The observed intermediary sporulation phenotype occurs when the QTL on chromosome XIV is homozygous at the AA1973 locus and the $R M E 1$ locus is either heterozygous or homozygous at the W303 allele, while the low sporulation phenotype occurs only when the loci on both chromosomes XIV and VII are homozygous at the AA1973 alleles. This would in turn result in the observed 12:3:1 phenotypic ratio of the $F_{2}$ generation as well as the observed 2:1:1 phenotypic ratio of the $\mathrm{Bc}_{1.2}$ generation.

\section{Molecular pathways controlling sporulation efficiency in yeast}

The effect of dominant epistasis has been previously reported to play a role in determining phenotypes of various traits in different organisms (20-22) and the significance of epistatic interactions is often vital in establishing the phenotypic variation observed in complex traits (2328). However, molecular mechanisms underlying quantitative trait interactions can be very difficult to discern (29), but given our results, we suggest a possible explanation of the observed epistatic interactions, integrating the phenotypic effects of loci on chromosomes XIV and VII (Fig. 4). The proteins produced by these loci most likely do not interact directly (16), but it is possible that their phenotypic effects converge in the signalling pathway, which leads to the initiation and realisation of the sporulation process (Fig. 4). The SWS2 and MKT1 genes of chromosome XIV are involved in mitochondrial function $(30,31)$ and could be linked with the respiration signal needed to successfully initiate and complete the sporulation process (32). Polymorphisms in the MKT1 gene affect the stability of the mitochondrial genome (31), while SWS2 codes for a mitochondrial ribosomal protein (33). These loci possibly promote high sporulation efficiency in the W303 strain, which could be due to the fact that they indirectly support more efficient respiration in the cell. Respiratory metabolism is central in initiating meiosis (34) and is required for providing the energy needed to complete the sporulation process (32). Thus the respiration signal of the W303 genes may be so efficient that it overrides the repressing effects of the $R M E 1$ gene, resulting in the observed DE inheritance. The convergence of the respiration signal and the effects of the RME1 gene on sporulation efficiency most likely takes place within the regulatory network of the main inducers of sporulation, the IME1 and IME2 genes (Fig. 4), whose activation (and subsequent entry into meiosis) can be achieved by the absence of the Rme1 protein (35) but also by a respiration-sensitive sig-

a)

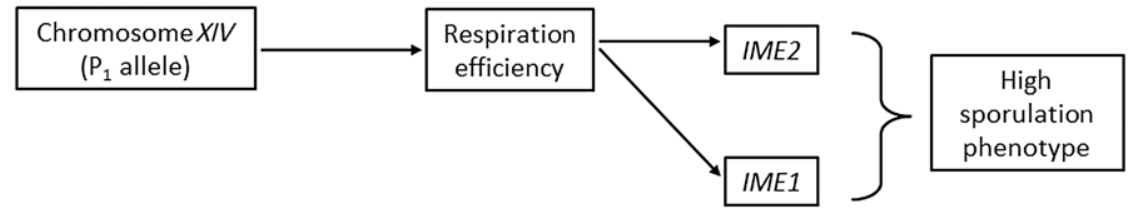

b)

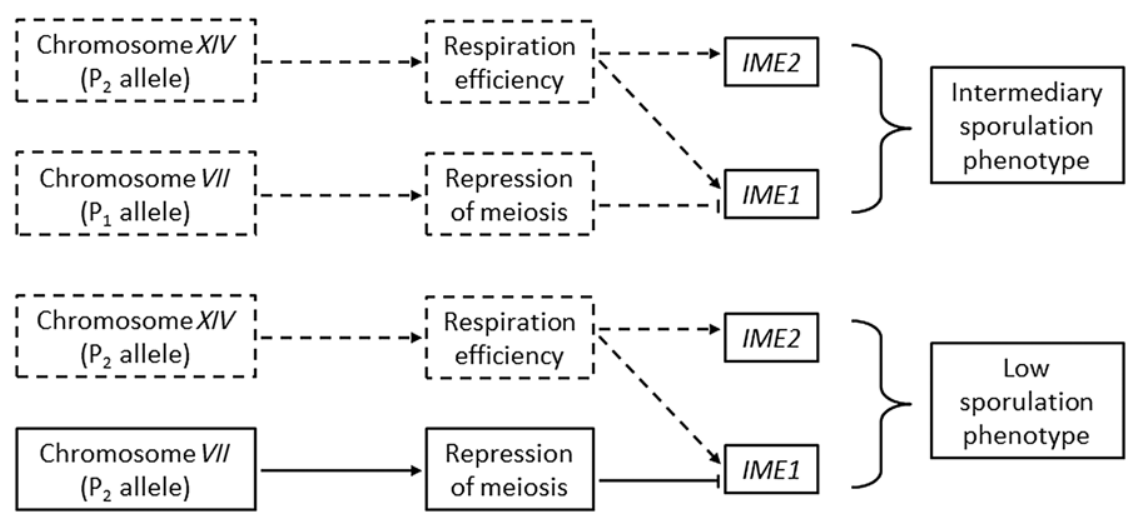

Fig. 4. Molecular pathways controlling sporulation efficiency in yeast. The solid/dashed lines represent interaction pathways leading to efficient/inefficient cellular respiration and efficient/inefficient repression of meiosis: a) whenever the chromosome XIV quantitative trait locus (QTL) contains the $\mathrm{P}_{1}$ (W303) allele (either in homozygous or heterozygous form), cellular respiration is efficient and sporulation efficiency is high. This is the dominant epistatic allele which masks the effects of the chromosome VII QTL, b) when the chromosome XIV QTL is homozygous for the $\mathrm{P}_{2}$ (AA1973) allele and the chromosome VII QTL is either homozygous or heterozygous for the $P_{1}$ allele, then an intermediary sporulation phenotype is expressed. This intermediary phenotype is characterized by inefficient cellular respiration and inefficient repression of meiosis, and c) when both QTLs are homozygous for the $\mathrm{P}_{2}$ alleles then the low sporulation phenotype is expressed. This phenotype is characterised by inefficient cellular respiration and efficient repression of meiosis 
nalling pathway (32). Furthermore, the PMS1 gene codes for an ATP-binding protein required for mismatch repair $(36,37)$ while the $F K H 2$ gene codes for a transcription factor involved in cell-cycle regulation (38). These genes also probably contribute to the variation in sporulation efficiency between the W303 and AA1973 strains but are not closely linked to respiration efficiency and were therefore excluded from the molecular pathway model (Fig. 4). However, in order to test the validity of this pathway model, further analyses based on nucleotide exchange methods are required.

This study supports the fact that single nucleotide differences can have a profound impact on the phenotypic variation of quantitative traits (5-7) and shows that single nucleotide differences could cause epistatic interactions between QTLs. In this particular case, the quantitative trait nucleotides (QTNs) most likely associated with the observed DE inheritance are probably the previously reported QTNs of the MKT1 coding region and SWS2 promoter region $(6,7)$ and the newly discovered QTNs of the RME1, FKH2 and SWS2 coding regions (Table 6). This study provides one of the most detailed views into the epistatic relationships of QTLs at the nucleotide level supplemented with a possible mechanistic explanation of the observed phenotypic distributions (Fig. 4). It is also important to note that the number of QTLs affecting sporulation efficiency of the two parental strains is probably higher than the observed number of QTLs, which is reflected by the significant Kolmogorov-Smirnov statistic of the two-locus DE1 model (Table 5). However, these loci are most likely small-effect QTLs. Studying the phenotype of a quantitative trait is a process that demands different approaches (39-42). Currently, the most widely used methods are based on whole genome sequencing and QTL-mapping (5-7) while the classical quantitative genetic analysis $(9,10)$, applied in this study, is becoming a less frequently used approach for studying such traits. In this paper we show that coupling biometrics with a computational approach can be a powerful means of providing additional insights into QTL interactions and mechanisms which regulate the phenotypic variation of quantitative traits. The six generation approach offers a good foundation for building theoretical inheritance models of such traits, using a simple analysis of phenotypic means and variances. The observed phenotypic distributions of these generations can be reproduced using computational modelling to generate theoretical phenotypic distributions by manipulating phenotypic values, frequencies and variances. This allows for the various statistical tests to be applied. Moreover, simulation-based strategies have long been applied in investigating quantitative traits (43-48), emphasising the role of computer modelling as a valuable aid in the analysis of such traits.

Finally, recent contemplation on the subject has emphasized the need for novel approaches in the field of quantitative genetics (49). Accordingly, our findings show that developing new methods is crucial for expanding our understanding of quantitative trait inheritance, providing a shift from the current research paradigm (centred mainly around large-scale genomics) towards reconciling classical quantitative genetics with genome-wide mapping studies. Altogether, this study shows that the complexity of studying quantitative traits can be counteracted by combining classical quantitative genetic with simulation-based, computational approaches, making possible the discovery of molecular mechanisms controlling phenotypic variation.

\section{Conclusion}

In this paper we have combined classical quantitative genetic analysis with phenotype modelling, a method that was developed for the purpose of this work, in order to analyse the variation in yeast sporulation efficiency. Such novel approach enabled us to obtain a detailed picture of the QTL relationships underlying the observed phenotypic variation. We have shown that the responsible loci shape the phenotype in dominant epistatic manner. Based on the molecular data, we propose that the observed genetic relationship arise from the interaction of multiple QTNs (all part of 60-kb long QTL region on chromosome XIV) and the RME1 locus on chromosome VII.

\section{Acknowledgements}

The SK1 and S288c strains were kindly provided by Prof. Dr. Sc. Franz Klein (Max F. Perutz Laboratories, Vienna, Austria) and the parental and $F_{1}$ generations of the W303/AA1973 strains were constructed prior to this study by Francis Fabre (Institute Curie, Orsay, France) and PTM (then Laboratory for Biology and Microbial Genetics, Faculty of Food Technology and Biotechnology, University of Zagreb, Croatia). We would like to acknowledge Prof. Franz Klein for providing necessary assistance as well as valuable discussions which helped to shape the whole project and Dr. Sc. Mary Sopta for critical reading of the manuscript. This work was supported by the Croatian Ministry of Science (grant number 098-0982913-2867).

\section{References}

1. Herskowitz I. Life cycle of the budding yeast Saccharomyces cerevisiae. Microbiol Rev. 1988;52:536-53.

2. Neiman AM. Ascospore formation in the yeast Saccharomyces cerevisiae. Microbiol Mol Biol Rev. 2005;69:565-84. http://dx.doi.org/10.1128/MMBR.69.4.565-584.2005

3. Mortimer RK. Evolution and variation of the yeast (Saccharomyces) genome. Genome Res. 2000;10:403-9. http://dx.doi.org/10.1101/gr.10.4.403

4. Gerke JP, Chen CT, Cohen BA. Natural isolates of Saccharomyces cerevisiae display complex genetic variation in sporulation efficiency. Genetics. 2006;174:985-97. http://dx.doi.org/10.1534/genetics.106.058453

5. Gerke JP, Lorenz K, Cohen BA. Genetic interactions between transcription factors cause natural variation in yeast. Science. 2009;323:498-501. http://dx.doi.org/10.1126/science.1166426

6. Deutschbauer AM, Davis RW. Quantitative trait loci mapped to single-nucleotide resolution in yeast. Nat Genet. 2005;37: 1333-40. http://dx.doi.org/10.1038/ng1674

7. Ben-Ari G, Zenvirth D, Sherman A, David L, Klutstein M, Lavi U, Hillel J, Simchen G. Four linked genes participate in controlling sporulation efficiency in budding yeast. PLoS Genet. 2006;2:e195. http://dx.doi.org/10.1371/journal.pgen.0020195 
8. Sherman F, Fink GR, Lawrence CW. Methods in yeast genetics. Cold Spring Harbor, NY, USA: Cold Spring Harbor Laboratory; 1979.

9. Kearsey MJ, Pooni HS. The genetical analysis of quantitative traits. Cheltenham, UK: Stanley Thornes Ltd; 1998.

10. Khodambashi M, Bitaraf N, Hoshmand S. Generation mean analysis for grain yield and its related traits in lentil. J Agr Sci Tech. 2012;14:609-16.

11. Simchen G, Jinks JL. The determination of dikaryotic growth rate in the basidiomycete Schizophyllum commune: A biometrical analysis. Heredity. 1964;19:629-49. http://dx.doi.org/10.1038/hdy.1964.75

12. Simchen G. Monokaryotic variation and haploid selection in Schizophyllum commune. Heredity. 1966;21:241-63. http://dx.doi.org/10.1038/hdy.1966.21

13. Simchen G, Connolly V. Changes in recombination frequency following inbreeding in Schizophyllum. Genetics. 1968;58: 319-26.

14. The $\mathrm{R}$ project for statistical computing, $\mathrm{R}$ software v. 3.1.2, The $\mathrm{R}$ foundation, Vienna, Austria; 2002. Available from: http://www.r-project.org.

15. Falconer DS, MacKay TF. Introduction to quantitative genetics. Harlow, UK: Pearson Education Limited; 1996.

16. The Web Primer tool, Saccharomyces Genome Database, Stanford University, Stanford, CA, USA. Available from: http://www.yeastgenome.org.

17. DNA Baser Sequence Assembler, v. 4, Heracle BioSoft, Piteşti, Romania; 2014. Available from: http://www.dnabaser.com.

18. Python ${ }^{\mathrm{TM}}$, v. 2.7, Python Software Foundation, Beaverton, OR, USA; 2001. Available from: http://www.python.org.

19. Blumental-Perry A, Li W, Simchen G, Mitchel AP. Repression and activation domains of Rme1p structurally overlap, but differ in genetic requirements. Mol Biol Cell. 2002;13:1709-21. http://dx.doi.org/10.1091/mbc.01-09-0468

20. Payne LN, Pani PK. Dominant epistatic gene which inhibits cellular susceptibility to RSV (RAV-O). J Gen Virol. 1971;13: 455-62. http://dx.doi.org/10.1099/0022-1317-13-3-455

21. Law CN, Sutka J, Worland AI. A genetic study of day-length response in wheat. Heredity. 1978;41:185-91. http://dx.doi.org/10.1038/hdy.1978.87

22. Adamkewicz L, Castagna M. Genetics of shell color and pattern in the bay scallop Argopecten irradians. J Hered. 1988; 79:14-7.

23. Fijneman RJ, de Vries SS, Jansen RC, Demant P. Complex interactions of new quantitative trait loci, Sluc1, Sluc2, Sluc3, and Sluc4, that influence the susceptibility to lung cancer in the mouse. Nat Genet. 1996;14:465-7. http://dx.doi.org/10.1038/ng1296-465

24. Long AD, Mullaney SL, Mackay TFC, Langley CH. Genetic interactions between naturally occurring alleles at quantitative trait loci and mutant alleles at candidate loci affecting bristle number in Drosophila melanogaster. Genetics. 1996; 144:1497-510.

25. Li Z, Pinson SR, Park WD, Paterson AH, Stansel JW. Epistasis for three grain yield components in rice (Oryza sativa L.). Genetics. 1997;145:453-65.

26. Routman EJ, Cheverud JM. Gene effects on a quantitative trait: two-locus epistatic effects measured at microsatellite markers and at estimated QTL. Evolution. 1997;51:1654-62. http://dx.doi.org/10.2307/2411217

27. Shook DR, Johnson TE. Quantitative trait loci affecting survival and fertility-related traits in Caenorhabditis elegans show genotype-environment interactions, pleiotropy and epistasis. Genetics 1999;153:1233-43.

28. Morel L, Croker BP, Blenman KR, Mohan C, Huang G, Gilkeson G, Wakeland EK. Genetic reconstitution of systemic lu- pus erythematosus immunopathology with polycongenic murine strains. Proc Natl Acad Sci USA. 2000;97:6670-5. http://dx.doi.org/10.1073/pnas.97.12.6670

29. Carlborg Ö, Haley CS. Epistasis: too often neglected in complex trait studies? Nat Rev Genet. 2004;5:618-25. http://dx.doi.org/10.1038/nrg1407

30. Huh WK, Falvo JV, Gerke LC, Carroll AS, Howson RW, Weissman JS, O'Shea EK. Global analysis of protein localization in budding yeast. Nature. 2003;425:686-91. http://dx.doi.org/10.1038/nature02026

31. Dimitrov LN, Brem RB, Kruglyak L, Gottschling DE. Polymorphisms in multiple genes contribute to the spontaneous mitochondrial genome instability of Saccharomyces cerevisiae S288C strains. Genetics. 2009;183:365-83. http://dx.doi.org/10.1534/genetics.109.104497

32. Jambhekar A, Amon A. Control of meiosis by respiration. Curr Biol. 2008;18:969-75. http://dx.doi.org/10.1016/j.cub.2008.05.047

33. Gan X, Kitakawa M, Yoshino K, Oshiro N, Yonezawa K, Isono $\mathrm{K}$. Tag-mediated isolation of yeast mitochondrial ribosome and mass spectrometric identification of its new components. Eur J Biochem. 2002;269:5203-14. http://dx.doi.org/10.1046/j.1432-1033.2002.03226.x

34. Piekarska I, Rytka J, Rempola B. Regulation of sporulation in the yeast Saccharomyces cerevisiae. Acta Biochim Pol. 2010; 57:241-50.

35. Mitchell AP, Herskowitz I. Activation of meiosis and sporulation by repression of the RME1 product in yeast. Nature. 1986;319:738-42. http://dx.doi.org/10.1038/319738a0

36. Williamson MS, Game JC, Fogel S. Meiotic gene conversion mutants in Saccharomyces cerevisiae. I. Isolation and Characterization of pms1-1 and pms1-2. Genetics. 1985;110:609-46.

37. Hall MC, Shcherbakova PV, Kunkel TA. Differential ATP binding and intrinsic ATP hydrolysis by amino-terminal domains of the yeast Mlh1 and Pms1 proteins. J Biol Chem. 2002;277:3673-9.

http://dx.doi.org/10.1074/jbc.M106120200

38. Zhu G, Spellman PT, Volpe T, Brown PO, Botstein D, Davis TN, Futcher B. Two yeast forkhead genes regulate the cell cycle and pseudohyphal growth. Nature. 2000;406:90-4. http://dx.doi.org/10.1038/35017581

39. Darvasi A. Experimental strategies for the genetic dissection of complex traits in animal models. Nat Genet. 1998;18:1924.

http://dx.doi.org/10.1038/ng0198-19

40. Mackay TF. Quantitative trait loci in Drosophila. Nat Rev Genet. 2001;2:11-20. http://dx.doi.org/10.1038/35047544

41. Mauricio R. Mapping quantitative trait loci in plants: Uses and caveats for evolutionary biology. Nat Rev Genet. 2001;2: 370-81.

http://dx.doi.org/10.1038/35072085

42. Steinmetz LM, Sinha H, Richards DR, Spiegelman JI, Oefner PJ, McCusker JH, Davis RW. Dissecting the architecture of a quantitative trait locus in yeast. Nature. 2002;416:326-30. http://dx.doi.org/10.1038/416326a

43. Hansen TF, Wagner GP. Modeling genetic architecture: a multilinear theory of gene interaction. Theor Popul Biol. 2001;59:61-86. http://dx.doi.org/10.1006/tpbi.2000.1508

44. Broman KW. Mapping quantitative trait loci in the case of a spike in the phenotype distribution. Genetics. 2002;163:116975.

45. Hermisson J, Hansen TF, Wagner GP. Epistasis in polygenic traits and the evolution of genetic architecture under stabi- 
lizing selection. Am Nat. 2003;161:708-34.

http://dx.doi.org/10.1086/374204

46. Doebeli M, Blok HJ, Leimar O, Dieckmann U. Multimodal pattern formation in phenotype distributions of sexual populations. Proc Biol Sci. 2007;274:347-57.

http://dx.doi.org/10.1098/rspb.2006.3725

47. Lettre G, Lange C, Hirschhorn JN. Genetic model testing and statistical power in population-based association studies of quantitative traits. Genet Epidemiol. 2007;31:358-62.

http://dx.doi.org/10.1002/gepi.20217

48. Rockman MV. Reverse engineering the genotype-phenotype map with natural genetic variation. Nature. 2008;456:738-44. http://dx.doi.org/10.1038/nature07633

49. Nelson RM, Pettersson ME, Carlborg Ö. A century after Fisher: time for a new paradigm in quantitative genetics. Trends Genet. 2013;29:669-76. http://dx.doi.org/10.1016/j.tig.2013.09.006 